\title{
Interplanetary Magnetic Field (IMF) control over the global structures of laboratory magnetosphere
}

\author{
Wei-Tsung $\mathrm{Fu}^{1, *}$, Gung Yur $^{2}$, Chi-Kuang Chao ${ }^{1}$, and Hafiz-Ur Rahman ${ }^{3}$ \\ ${ }^{1}$ Institute of Space Science, National Central University, Taoyuan City, Taiwan \\ ${ }^{2}$ Air Asia Co. LTD., Tainan Airport, Tainan City, Taiwan \\ ${ }^{3}$ Department of Physics, University of California, Irvine, California, U.S.A.
}

\section{Article history: \\ Received 10 January 2019 \\ Revised 27 May 2019 \\ Accepted 16 June 2019 \\ Keywords: \\ Laboratory simulation, \\ Magnetosphere, Magnetotail \\ Citation:}

Fu, W.-T., G. Yur, C.-K. Chao, and H.-U. Rahman, 2020: Interplanetary Magnetic Field (IMF) control over the global structures of laboratory magnetosphere. Terr. Atmos. Ocean. Sci., 31, 49-60, doi: 10.3319/ TAO.2019.06.16.02

\begin{abstract}
Both east-west and north-south view images of magnetosphere for various interplanetary magnetic field (IMF) parameter have been investigated simultaneously in a laboratory experiment of the solar wind-magnetosphere coupling. An aurora is observed for both case of southward and northward IMF but the luminosity becomes stronger for southward IMF. The $\mathrm{X}$ type reconnections in the front and tail areas for southward IMF clearly shown in the magnetosphere images for higher IMF magnitude. The distances between the terrella and the front side and tail reconnection for southward IMF are increased as the magnitude of IMF decreases. A sector of discontinuity boundary which corresponds to the reconnection area is observed on the tail region of the north-south view magnetosphere when the IMF is southward. With northward IMF applied, a long narrow plasma beam jet in the magnetotail has been observed for the east-west view while a water drop shape of magnetotail is shown in the north-south view. Inserting the 20 degree terrella tilt, the study of modeling analysis is also processed for comparison with photograph of the experimental results in the laboratory simulation. The OpenGGCM model on CCMC overview of the global structure, the comparisons show that the model presented lower temperature in the magnetotail for the northward IMF. This corresponds to the weak and diffused tail in the laboratory simulation. For the southward IMF, the model presented higher temperature in the magnetotail, which corresponds to the more luminous tail region in the laboratory simulation. And the curvature of the magnetic lines due to the dipole tilt is qualitatively matched.
\end{abstract}

\section{INTRODUCTION}

Plasma physics process includes situs observations, theoretical predictions, and laboratory investigations. Especially in space plasma physics, the theoretical models have played the most important role in the development of understanding space phenomena in the past years and laboratory experiments have made significant contributions as well. In order to develop space physics, many space missions are executed to obtain the situs data. However, the missions are still not common and because of the insufficient amount of the returned data, there are many mysteries even in fundamental properties of the solar wind interaction with the planets. After all, an understanding of the solar wind interaction

\footnotetext{
* Corresponding author

E-mail:mariofu@shsh.ylc.edu.tw
}

with the Earth dipole is essential to basic planet-topological problems. Therefore, a fully controllable laboratory simulation experiment is needed to be an attractive approach to study some of the fundamental problems related to the solar wind interaction with planets.

In history, Birkeland's experiment (Birkeland 1908) was a major advance for space physics 110 years ago. He used "terrella" laboratory experiments by using an electron beam streaming against a dipole-magnetic field to simulate the earth magnetosphere. Plasma beams were used from 1950s (Bostick et al. 1963; Cladis et al. 1964; Kawashima and Fukushima 1964; Kawashima and Mori 1965). For the last 50 years, many terrella experiments have been conducted by several groups (Podgorny and Sagdeev 1970; Podgorny 1976; Podgorny et al. 1978, 2003; Baum and 
Bratenahl 1982; Minami and Takeya 1985; Minami et al. 1993; Rana et al. 2004; Xiao et al. 2017). Briefly, the most important function of these plasma experiments is improving the interpretation of space data. However, plasma experiments have been responsible for major advances in understanding space plasmas.

There are three essential ingredients for the composition of Earth's magnetosphere in the laboratory simulation. The first ingredient is the solar wind, fully ionized hydrogen plasma that streams from the sun. The second is the Earth's magnetic field that has the same form as that of a bar magnet, but elliptical shape dipole magnetic field. The third ingredient, large-scale IMF, plays an important role in the Earth's interaction with the solar wind.

The interaction between the solar wind and the Earth's magnetic field was simulated by the terrella experiments in a laboratory plasma chamber at University of California, Riverside (UCR) T1. Applying with suitable scaling laws, magnetized plasma fluxes interacting with a magnetic dipole can achieve the laboratory simulating magnetosphere qualitatively. The flowing magnetized plasma beam interaction with a stationary dipole magnetic field is fabricated by the laboratory experiments at UCR T1 space simulation facility. The facility was upgraded in order to satisfy the scaling requirements (Rahman et al. 1991).

Although individual magnetospheric phenomena can be successfully reproduced and investigated in the laboratory, it is still hard to know the global structure. The main reason is that the exact scaling of the relevant parameter ratios from the planetary-magnetosphere to a laboratory experiment is unrealistic / impractical. However, these individual experiments still address the formation of each region of the magnetosphere in space physics. The tail and front regions have been studied at the UCR laboratory and observed for the southward and northward interplanetary magnetic fields (IMF) (Birn et al. 1992; Yur et al. 1999, 2012). Laboratory simulations performing magnetosphere-solar wind interactions have been continued by different groups (Horton and Chiu 2004; Horton et al. 2007; Brady et al. 2009; Shaikhislamov et al. 2011, 2014; Saitoh et al. 2015). The scaling laws they used are worth investigating although there are no IMFs in the laboratory simulations.

The East-west view of magnetosphere had been presented in both theoretical and experimental simulation papers (Rahman et al. 1991; Birn et al. 1992; Yur et al. 1995) while north-south view of the magnetosphere have not been fully investigated in the laboratory experimental simulation. In order to understand the global structure of the magnetosphere both east-west and north-south images are required to observe for in the first step. This paper present both the east-west and north-south views of laboratory simulated magnetosphere with various IMF parameters simultaneous. The influences of IMF on the global laboratory magnetosphere structure are studied from photographic images.
These studies are concentrated on the aurora, reconnection areas and magnetotail.

When the global features with the planetary-magnetosphere structures are first concerned, the highest strength $400 \mathrm{G} \mathrm{IMF}$ is applied and north-south view of chamber images is shown in this paper. Inserting the terrella tilt $20 \mathrm{de}$ gree and $400 \mathrm{G}$ magnitudes for northward and southward IMF, photograph images are also taken for comparison with the study of modeling analysis.

The laboratory simulation experiment and modeling is described in the following section 2. Section 3 presents the laboratory and modeling simulation results and followed by discussion and conclusion, sections 4 and 5 respectively.

Lack of budget support, the UCR-T1 laboratory simulation was terminated in 2002. The final images data of these experiments are shown in this paper before the laboratory shut down, and hopefully provide following researchers with a reference and guidance in the future.

\section{LABORATORY SIMULATION EXPERIMENT AND MODELING}

The simulation experiment consists of an intense magnetized plasma beam, produced by a large plasma gun, interacting with a strong dipole field to form a magnetosphere that scales to some degree of the earth's magnetosphere.

The UCR-T1 space simulation device is consists of a cylindrical vacuum chamber, $1.3 \mathrm{~m}$ in diameter and $11 \mathrm{~m}$ length. Outlook of the UCR space simulation test facility and inner view of interaction chamber are displayed in Fig. 1a. Including a test section made of filament wound fiberglass and a dump tank made of steel for the suppression of disturbing effects of shocks reflected back into the test section within the test period. Schematic of experimental simulation is presented in Fig. 1b. Schematic diagrams of the dipole field and IMF direction in the UCR-T1 laboratory simulation is shown in Fig. 1c. In the top panel, a southward IMF is applied as shown in the top panel and a northward IMF in the bottom panel. A typical time sequence of magnetic field intensities picked by a magnetic probe located near a terrella for each device is illustrated in Fig. 1d according to real oscillograph records.

The solar wind is simulated by a coaxial plasma gun operating in a deflagration mode. The electrodes of the gun are directly connected to a $10 \mathrm{kV}$ maximum energy source and hydrogen gas is injected to initiate the discharge. The gun is powered by a $450 \mu \mathrm{F}$ capacitor bank which is normally charged to $6.5 \mathrm{kV}$ delivering $141 \mathrm{kA}$ of current. The hydrogen gas is injected between the gun electrodes when a fast valve is opened electromagnetically by firing a $30 \mu \mathrm{F}$ capacitor bank charged to $5.3 \mathrm{kV}$. The hydrogen gas is discharged by the high electric field as a field emission process to form hydrogen plasma. A single shot produces a plasma pulse of about $100 \mu$ s duration flowing through the drift 
chamber to form magnetic field variations caused by the gun in the 3rd row and by the plasma in the 4th panel of the Fig. 1d. For the closely match the earth's dipole field, the terrella is made of a $5.5 \mathrm{~cm}$ diameter billiard ball arranged as a L-C transmission line, with spherically wound 10 turn coil embedded in epoxy powered by a $500 \mu \mathrm{F}$ capacitor bank charged to $5.3 \mathrm{kV}$ that delivers a dipole field up close to $27.5 \mathrm{kG}$ at the equator for about $200 \mu$ s period. The dipole magnetic field is typically shown in the 2nd panel of Fig. 1d and is generated by a high current caused by a discharge of the capacity bank to flow through the coil around the billiard ball surface according to Ampere law. However, it is difficult to suspend the terrella without a stand to wire the current coil. Inevitably, it is possible to block some plasma flow around the stand and observations near the bottom side of the terrella connected to the stand shall be interpreted carefully.
As the plasma beam flowing through the drift chamber with a flow velocity of about $4 \times 10^{7} \mathrm{~cm} \mathrm{~s}^{-1}$, it will be magnetized by IMF simulated by an external coil with the glued taped fixed attached with the test chamber. The vertical ( $\mathrm{z}$ axis) magnetic field with a maximum strength of $500 \mathrm{G}$ and a cycle period of $2 \mathrm{~ms}$ can be supplied.

The plasma flux is magnetized by IMF that is generated by coils attached outside the chamber of the test section. Magnetizing degree of the model solar wind depends on the beam fly time that plasma beam passes through the chamber and the applied magnetic field strength of IMF. It has been concluded the after propagating a distance of $3.75 \mathrm{~m}$ that is terrella located in the chamber, over the strength of 100 Gauss was necessary to obtain significant magnetization. The magnetic field caused by the IMF is shown in the top panel of Fig. 1d and is designed to last constant magnetic (a)

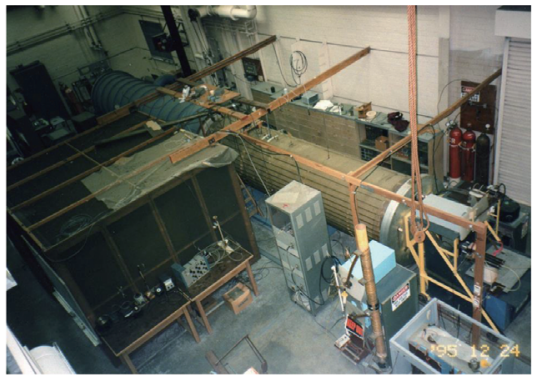

(b)

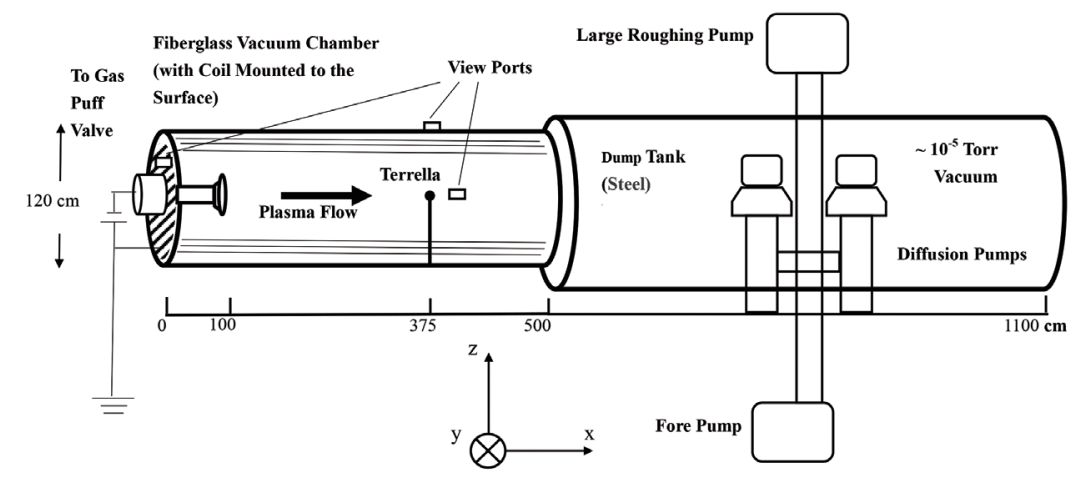

(c)

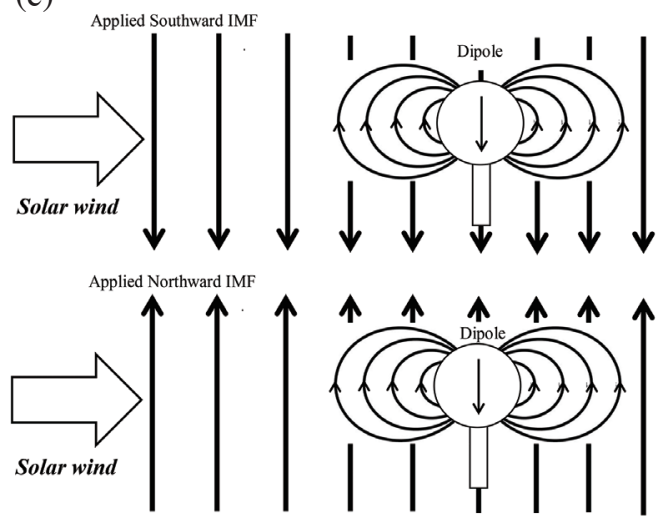

(d)

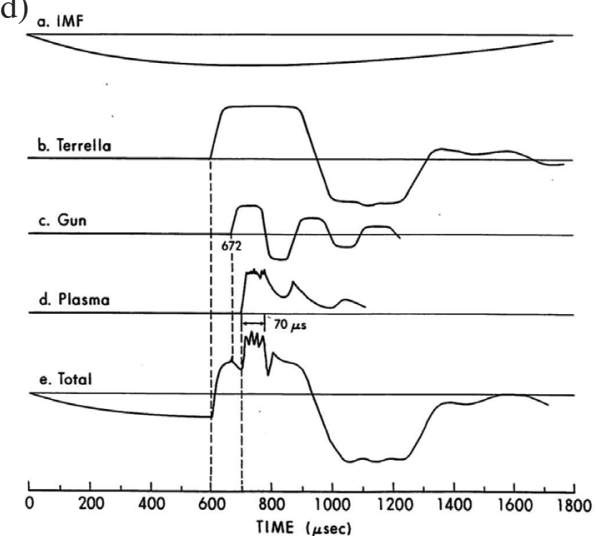

Fig. 1. (a) Outlook of of the UCR space simulation test facility (left) and inner view of interaction chamber (right). (b) Schematic of the laboratory experiment setup in simulation. (c) Schematic diagrams of the dipole field and IMF direction in the UCR-T1 laboratory simulation. (d) The oscillographs of IMF, terrella, gun, plasma, and total picked up by a magnetic probe and Rogowski coil. 
field intensity for more than $1 \mathrm{~ms}$. Magnetized beam leads to an $\overrightarrow{\mathrm{E}} \times \overrightarrow{\mathrm{B}}$ propagation with a slow diffusion rate, which can be considered as fully magnetized before interacting with the dipole field. The flow speed is super-Alfvénic for the most of the pulse duration.

The long range propagation of a plasma beam in a transverse magnetic field (IMF) is studied experimentally. Beam propagation at low magnetic fields is dominated by diamagnetic effects and by the magnetized $\overrightarrow{\mathrm{E}} \times \overrightarrow{\mathrm{B}}$ drift at high fields (Song et al. 1990; Wessel et al. 1990; Rahman et al. 1991). For the magnetized flow of the plasma beam (simulated solar wind) is established, the drift vacuum chamber $1.3 \mathrm{~m}$ in diameter and $11 \mathrm{~m}$ length in fiberglass material is necessary. A combined oscillograph of the IMF, terrella and the plasma pulse is shown in the bottom panel of Fig. 1d.

The plasma beam is collisionless that the mean free path $\lambda_{e i}$ is on the order of $1-2 \mathrm{~m}$ which is comparable to the characteristic scale size (Yur et al. 1995). The original plasma beam before interacting with terrella and other relevant parameters are presented in the earlier paper by Yur et al. (1995). The simulation satisfies most of the scaling situations except $\lambda_{A} / x_{0}\left(\lambda_{A}=2 \pi v_{A} / \omega_{i}\right.$ is the Alfvén wavelenrth and $x_{0}$ is the size of the magnetosphere) which need to be smaller than unity. In order to satisfy the condition $\lambda_{A} / x_{0}<<$ 1 , a higher density plasma for shorter $\lambda_{A}$ and stronger dipole field for longer $x_{0}$ are required. This can be achieved by increasing the voltages for plasma gun and field coils of generating IMF. This requires a significant improvement in experimental facility. Nevertheless, the results of the present experiment may be applicable to the large MHD scales because the ratio $r_{i L} / x_{0} \approx 0.1$ ( $r_{i L}$ is the ion gyroradius) is still somewhat smaller than unity. Our emphasis here is not to establish an exactly scaled laboratory model of the earth's magnetosphere but rather on a qualitative understanding of structures and processes.

The other two important dimensionless parameters are $\beta_{d}$ and $\beta_{p}$, which ensures that the Mach number is scaled the same scale as in space. Meanwhile the magnetic Reynolds number and Hall parameter are kept the same order as in space. The various parameters in space and the laboratory simulation experiment together with the required scaling parameters are listed in Table 1, and the practical results simulated at IMF equal to $400 \mathrm{G}$ are also presented. Magnetic field was measured by a magnetic probe and plasma density,

Table 1. Solar Wind and Earth Parameters (Yur et al. 1999).

\begin{tabular}{|c|c|c|c|}
\hline \multicolumn{4}{|c|}{ Measured Parameters } \\
\hline Magnetic field in solar wind $B_{\mathrm{IMF}}(\mathrm{G})$ & 200 & 300 & 400 \\
\hline Plasma density $n_{0},\left(\mathrm{~cm}^{-3}\right)$ & $7.6 \times 10^{12}$ & $19.8 \times 1^{012}$ & $32.9 \times 10^{12}$ \\
\hline Solar wind velocity $v_{0},\left(\mathrm{~cm} \mathrm{~s}^{-1}\right)$ & $1.3 \times 10^{7}$ & $1.2 \times 10^{7}$ & $1.1 \times 10^{7}$ \\
\hline Required magnetospheric size, $x_{0},(\mathrm{~cm})$ & & 10 & \\
\hline Magnetic field inside the plasma $B_{P},(G)$ & 50 & 180 & 320 \\
\hline Electron temperature $\mathrm{T}_{\mathrm{e}},(\mathrm{eV})$ & 15.6 & 18.3 & 20.7 \\
\hline Ion temperature $\mathrm{T}_{\mathrm{i}},(\mathrm{eV})$ & 3.12 & 3.66 & 4.02 \\
\hline \multicolumn{4}{|c|}{ Derived Parameters } \\
\hline Electron plasma frequency $\omega_{p e},\left(\mathrm{rad} \mathrm{s}^{-1}\right)$ & $1.6 \times 10^{11}$ & $2.5 \times 10^{11}$ & $3.2 \times 10^{11}$ \\
\hline Ion plasma frequency $\omega_{p i},\left(\operatorname{rad~s}^{-1}\right)$ & $3.7 \times 10^{9}$ & $5.9 \times 10^{9}$ & $7.6 \times 10^{9}$ \\
\hline Electron skin depth $c / \omega_{p e},(\mathrm{~cm})$ & 0.19 & 0.12 & 0.09 \\
\hline Ion inertia length $c / \omega_{p i},(\mathrm{~cm})$ & 8.3 & 5.1 & 4 \\
\hline Debye length $\lambda_{D},(\mathrm{~cm})$ & $1.1 \times 10^{-3}$ & $0.8 \times 10^{-3}$ & $0.5 \times 10^{-3}$ \\
\hline Collision mean free path $\lambda_{e i},(\mathrm{~cm})$ & 108.4 & 57.4 & 36.7 \\
\hline Electron gyroradius $r_{i L},(\mathrm{~cm})$ & 0.2 & 0.06 & 0.03 \\
\hline Alfven wave length $\lambda_{A},(\mathrm{~cm})$ & 51 & 31 & 24 \\
\hline Ion gyroradius $r_{i L},(\mathrm{~cm})$ & 3.9 & 1.14 & 0.86 \\
\hline Alfvén Mach number $M_{A}$ & 3.45 & 1.15 & 0.94 \\
\hline Dynamic beta $\beta_{D}=4 \pi \rho_{0} v_{0}^{2} / B_{0}^{2}=M_{A}^{2}$ & 12 & 1.32 & 0.88 \\
\hline Thermal beta $\beta_{P}=4 \pi n_{0} T_{0} / B_{0}^{2}$ & 1.15 & 0.27 & 0.15 \\
\hline Magnetic Reynolds number $R_{m}=R_{H}\left(\Omega_{e} \tau_{e i}\right)$ & 359 & 432 & 454 \\
\hline Hall parameter $R_{H}=\beta_{D}\left(x_{0} / r_{i L}\right)$ & 30 & 12 & 11 \\
\hline$r_{i L} / x_{0}$ & 0.4 & 0.11 & 0.08 \\
\hline$\lambda_{A} / x_{0}$ & 5 & 3 & 2 \\
\hline
\end{tabular}


solar wind, electron temperature, and ion temperature, were measured by a Faraday cup. The other parameters were derived from the measured parameters. The solar wind parameters were measured at position of $3.75 \mathrm{~m}$ without the terrella. The measuring time period was 70 - $100 \mu$ s that considered as the interaction time for IMF, dipole field and plasma beam as shown in Fig. 1d. The details of the experimental setup and scaling have been previously described in (Rahman et al. 1989, 1991; Birn et al. 1992; Yur et al. 1995, 2012).

In order to compare the laboratory simulation results to the realistic magnetosphere, the NASA's Community Coordinated Modeling Center (CCMC) Run-on-Request system (http://ccmc.gsfc.nasa.gov) is applied with supported several global magnetosphere simulations in literature. The global MHD models includes BATS-R-US (Block Adaptive Tree Solar-wind Roe Upwind Scheme) (Powell et al. 1999), GUMICS-4 (Grand Unified Magnetosphere-Ionosphere Coupling Simulation) (Janhunen et al. 2012), LFM (Lyon-Fedder-Mobarry) (Lyon et al. 2004), and OpenGGCM (Open General Geospace Circulation Model) (Raeder et al. 2001).

\section{EXPERIMENTAL AND MODELING RESULTS}

In this paper, full structures of the simulated magnetosphere under controlling the differently applied IMF are presented. The east-west view corresponds to the $\mathrm{X}-\mathrm{z}$ plane while the north-south view corresponds to the $x-y$ plane
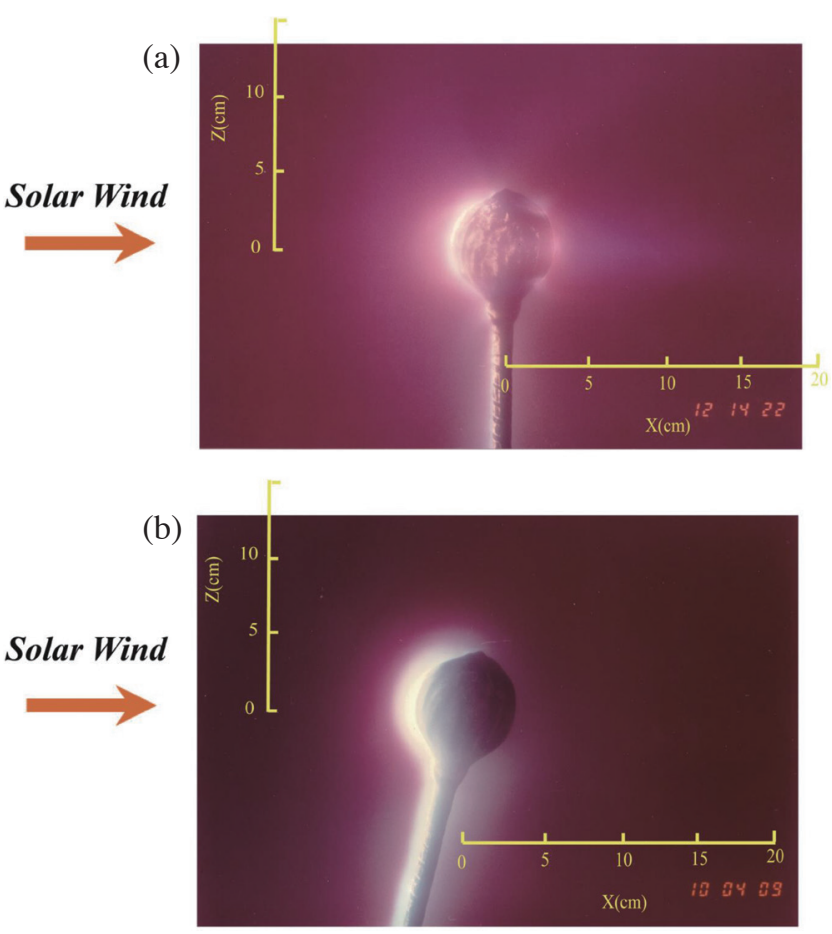

Fig. 2. (a) Photograph of the side-viewed simulated magnetosphere for 0 G IMF with dipole tilt 0 degree. (b) Photograph of the side-viewed simulated magnetosphere for southward IMF of $400 \mathrm{G}$ with tilt angle 20 degree, but no dipole field. in Geocentric Solar Magnetospheric (GSM) coordinate. Figure 2a shows the photograph of the east-west view with $0 \mathrm{G}$ IMF and no tilt terrella, the un-magnetized plasma beam first stopped by the dipole field and then hit the surface of terrella. Figure $2 b$ shows the photograph of east-west view with $400 \mathrm{G}$ IMF and tilt 20 degree terrella but no dipole field at all. Without the interaction between the magnetized plasma and the dipole, the global structure of magnetosphere can't be seen. Obviously, the light of terrella at the front side only caused by the plasma beam hit and evaporated the epoxy on the surface of terrella.

In order to view the front and tail region more clearly, the magnitude IMF increased to $400 \mathrm{G}$ and meanwhile less contrast films have been used for the following photographs. Only one camera was used for zooming in the east-west view to show the fine global structures in the Fig. 3, and the position of the terrella was at the center of the photos. Under taking optical images, clearly shown topological structures of the simulated Earth's magnetosphere are presented at terrella tilt angle of zero degree. Figures $3 a$ and $b$ show the clearly global structure of magnetosphere in east-west view for increasing the magnitude of IMF into $400 \mathrm{G}$ that is ever not presented before. The reconnections in the front region and an extended X-type structure in the tail region are able to be clearly identified in Fig. 3a for $400 \mathrm{G}$ southward IMF. For $400 \mathrm{G}$ northward IMF in Fig. 3b, we observe a transition from a broader plasma sheet originating from the terrella to a
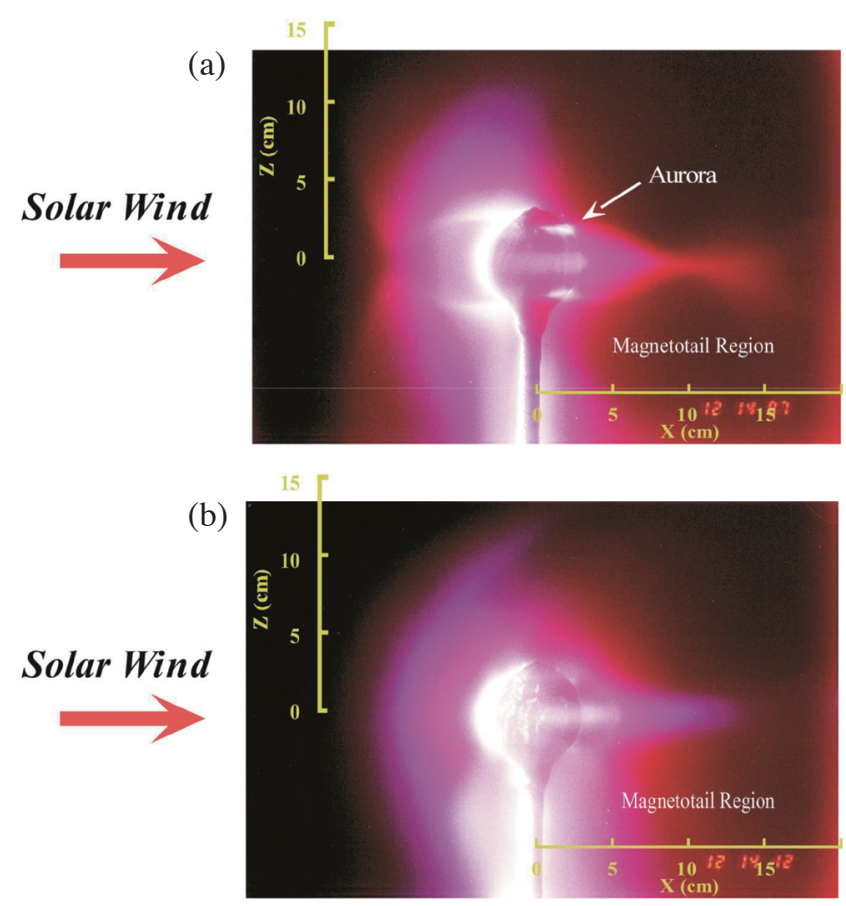

Fig. 3. (a) Photograph of the east-west viewed simulated magnetosphere for southward IMF of $400 \mathrm{G}$, showing an apparent aurora zones, and the near-Earth tail region. (b) Photograph of the east-west viewed simulated magnetosphere for northward IMF of $400 \mathrm{G}$, showing an apparent the near-Earth tail region. 
much narrower sheet further down tail, which is indicative of a Y-type magnetic neutral line. These results are explained by Birn (Birn et al. 1992), which are consistent with the previous magnetic field measurements in the tail region of the model magnetosphere. It is noted that the magnetospheric size is defined as the distance from the center of terrella to the front edge between bright and dark area on the photos.

By using two monocular cameras with open shutter put in different axes view ports of plasma chamber to obtain time integrated photographs in a UCR-T1 laboratory experiment of the solar wind- magnetosphere coupling. Both zoomed out to obtain panoramic views, the position of the terrella was closed to the left of the center in the photos. Both east-west and north-south view images of magnetosphere for 200, 300, and $400 \mathrm{G}$ magnitudes of the southward and northward IMF are investigated simultaneously. Figures $4 \mathrm{a}$ and $\mathrm{b}$ present the images of the laboratory magnetosphere with (1) 200 , (2) 300 , and (3) 400 Gauss southward IMFs for east-west and north-south view respectively. A bright belt at the equator areas, which might correspond to the Van-Allen radiation belt, is presented. A circular aurora and a radial type of magnetotail are shown in the northsouth view images. It can be seen clearly that the aurora at high latitude occurred on the surface of the terrella, while the bright belt near the equator is on the outside of the terrella. The mechanism of the aurora in the laboratory is not exactly the same as in the atmosphere. Although it was considered as the impact by the high-energy particles from the tail reconnection, in the laboratory, the light was emitted from the epoxy on the surface of the terrella, which is different from the emission by gas in the atmosphere on the earth.

The aurora is brighter at the dusk area and faints at the dawn area. The plasma intensity is lower at the midnight than the dawn-dusk area of magnetotail in the northsouth view images. This suggests that the reconnection is all around the terrella and the density of the plasma sheet is larger at the dawn-dusk area than in the midnight area. The distance of the reconnection point is varied with the geometry position. The position of the reconnection is closer to the terrella at dawn-dusk area $(\approx 10 \mathrm{~cm}$ for IMF $=400 \mathrm{G}$ on the photograph) than in the midnight area $(\approx 16 \mathrm{~cm})$.

Figures 5a - c present similar images for 200, 300, and $400 \mathrm{G}$ northward IMFs respectively. A bow shock, the plasma mantle and a comet like long narrow tail are clearly shown in the east-west view images. The plasma intensity is increased as IMF magnitude increased. A faint aurora with a brighter section near midnight area is shown in the northsouth images. The magnetotail becomes water drop shape as IMF increases from 200 to $400 \mathrm{G}$. The magnetotail is asymmetric and shifts to the dawn side. Two plasma streams are shown on the dusk area. The outer one might correspond to the bow shock while the inner one to the long narrow tail.

The location of the aurora is dependent on the beam drift, it is caused that the magnetized plasma flux is fol- lowed by $\overrightarrow{\mathrm{E}} \times \overrightarrow{\mathrm{B}}$ propagation. The $\overrightarrow{\mathrm{E}} \times \overrightarrow{\mathrm{B}}$ propagation is accompanied by beam compression transverse $\mathrm{B}$ with as much as a factor of four increases in density and a drift of the beam in the direction of the ion Lorentz force. Due to the deflection of plasma beam propagation is followed by the ion Lorentz force direction. The dark areas of the downer left corner of photograph is increase as the magnitude of the applied southward IMF is increased in Fig. 4 and the dark areas of the upper left corner of photograph are increase as the applied northward IMF is increase in Fig. 5.

The front side and tail reconnections are clearly showing in the southward and northward IMF, the curvature of the front reconnection is increased as the magnitude of the applied IMF is increased. The photographs also show that the distances between the center of terrella and the reconnection areas are decreased as the applied IMF magnitude increases. Figure 6 presents the reconnection distances directly measured from the photographs by a ruler for various IMFs in the front region and the error bars were made by visual determination. The magnetospheric size that distance from the magnetopause to the center of terrella, is between 10 and $13 \mathrm{~cm}$ depends on the applied IMF and solar wind parameters. The magnetospheric size becomes smaller for the stronger IMF, higher solar wind velocity and density.

Figure 7 presents the global structure of magnetosphere in east-west view with 20 degree dipole tilt. In Fig. 7a, the image of southward IMF of $400 \mathrm{G}$ is shown. The front side reconnection can be clearly identified and the tail reconnection is faint. The twisted magnetotail, merged under the solar wind and tilted dipole magnetic field, can be also observed, which is merged the aurora zones are apparent but the intensity is lower. The $\mathrm{X}$ point in the front side moves northward, and the curvature is not as obvious as without dipole tilt. In Fig. 7b, the magnetosphere structure of northward IMF $400 \mathrm{G}$ is also shown. The twisted comet tail can be seen but there are no narrow long tails at the further down tail, and the intensity is lower as well. Near the equator in the tail region disappear the brighter areas.

\section{DISCUSSION}

The laboratory simulations of solar wind/magnetosphere interaction at the UCR space simulation facility is used to investigate the domination of IMF on the global structures of the model magnetosphere. Both east-west and north-south images are presented and studied. The current regions in the magnetosphere are typically considered as a region of hot plasma. The hot plasma interacts with the neutral background gas and permits us to obtain global images of the model magnetosphere that show primarily the embedded current sheets. The images of the magnetopause, the aurora, the reconnection area, and the magnetotail are clearly shown. The plasma intensity increases as the magnitude of IMF increases because IMF confines plasma. The observed 

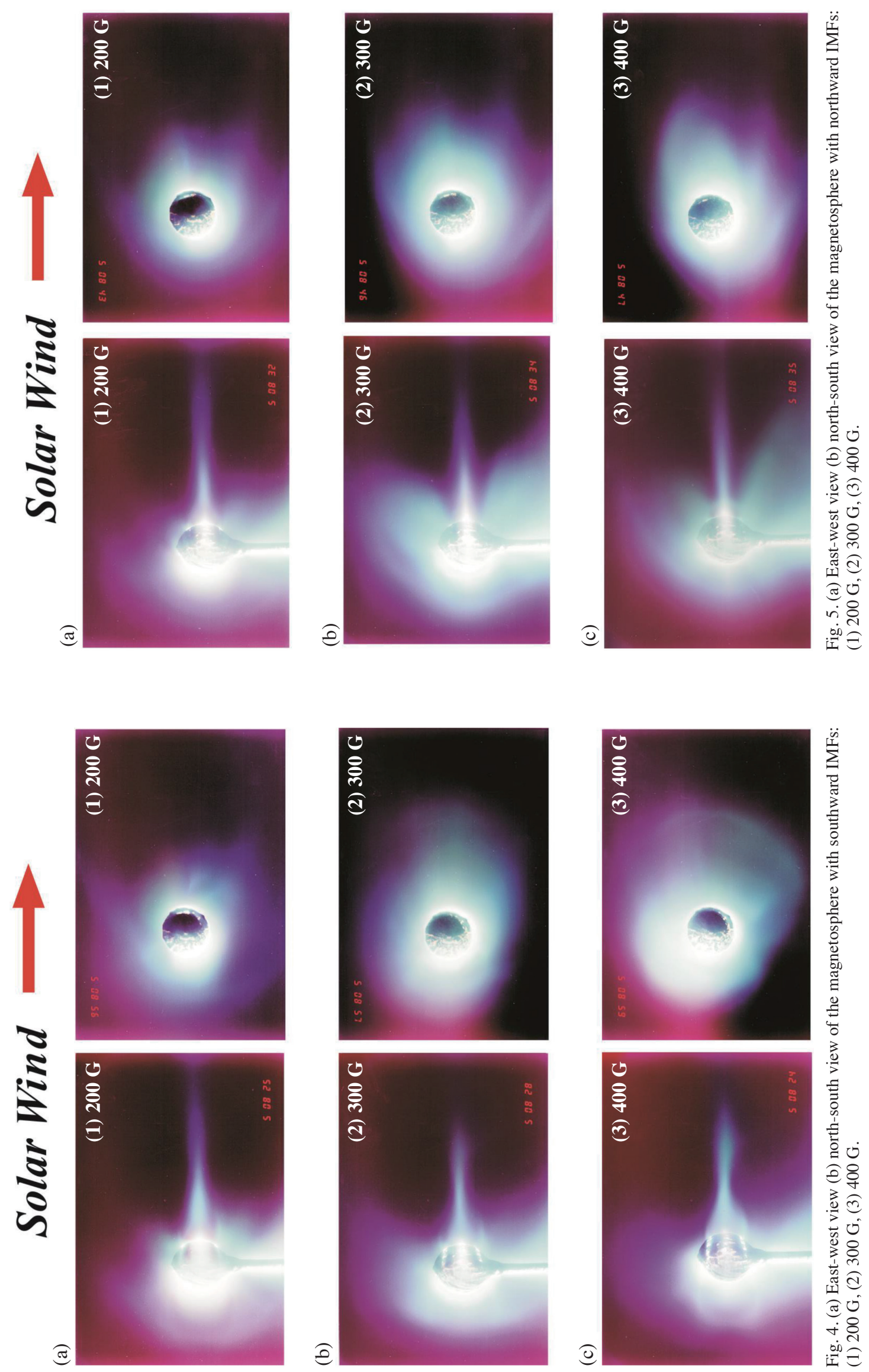


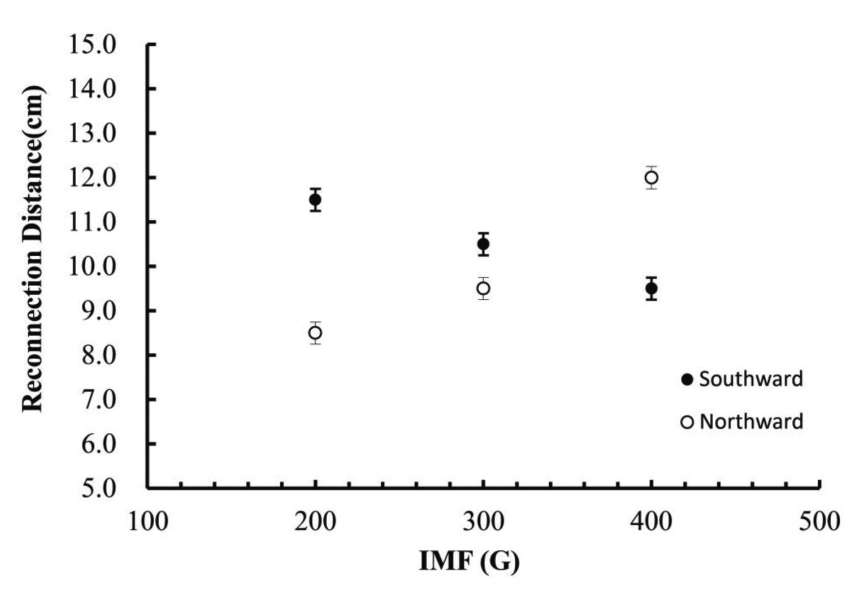

Fig. 6. Reconnection distances versus various IMFs in the front region.

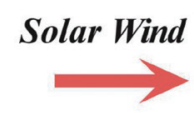

(a)

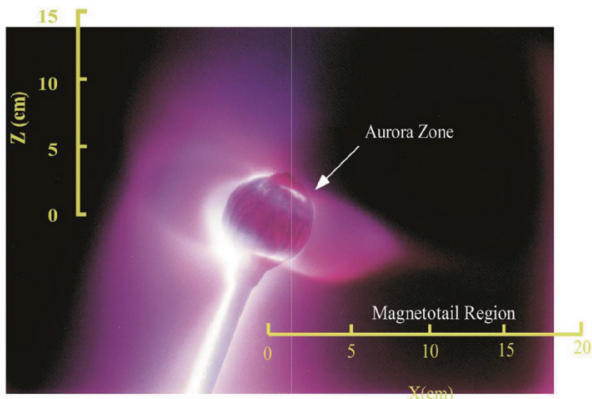

(b)

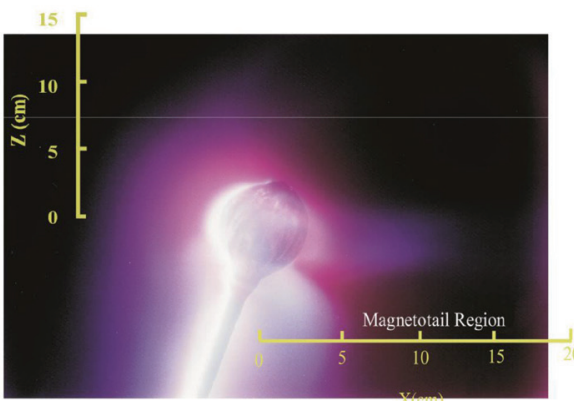

Fig. 7. (a) Photograph of the simulated magnetosphere (dipole tilt angle of 20 degrees) for southward IMF of $400 \mathrm{G}$, showing an apparent aurora zones, and the near-Earth tail region. (b) Photograph of the simulated magnetosphere (dipole tilt angle of $\sim 20$ degrees) for northward IMF of $400 \mathrm{G}$, showing an apparent the near-Earth tail region.

aurora is produced by the impaction of earthward plasma stream along the edge field line of near earth plasma sheet of field aligned current (FAC) on the surface of the terrella. The aurora luminosity is higher for southward IMF than that for northward IMF, which is coincident with the actual phenomena (Craven et al. 1991; Oka et al. 2018).

In the tail region, the ion flux and electron flux are combined to produce the net current density. The currents are dominantly carried by electrons. The currents are streaming tailward between $\mathrm{z}=0$ and $3 \mathrm{~cm}$ (the plasma sheet region), and then reversed to earthward for $\mathrm{z}=3$ to $11 \mathrm{~cm}$ (the lobes and mantle) (Yur et al. 1999). It suggests that the observed aurora is produced by the impaction of earthward plasma stream along the edge field line of the near earth plasma sheet of the FAC on the surface of the terrella.

Connection of FAC in the ionosphere shows that the FAC is generated in the magnetotail due to the earthward electric field appearance (Birn and Hesse 1996; Birn et al. 1997; Podgorny et al. 2003). The electric field in MHD approximation can be expressed by $\vec{E}=-\vec{v} \times \vec{B} / c+\vec{j} \times \vec{B} / n e c+\nabla p_{e} / n e$. The $\nabla p_{e}$ term is negligible. The velocity is directed to Sun, and the electric field directed to Sun can be only the Hall electric field $\vec{j} \times \vec{B} /$ nec. The plasma flow is also accelerated toward the Earth by $\overrightarrow{\mathrm{j}} \times \overrightarrow{\mathrm{B}} /$ nec force. A westward electrojet is produced near the terrella surface region to connect the circuit. Both north-south magnetosphere images for southward and northward IMF are asymmetric in the experimental simulation. They show more intense plasma at the dusk area and the aurora is faint at the dawn area. This can be explained by the westward electrojet, especially the electrons flows toward the dusk area. The aurora luminosity is higher for southward IMF than that for northward IMF, which is coincident with the earthward beam is stronger when IMF is southward.

Another significant result of distant magnetotail is the bright belt near the equator region. This region is similar to the Van-Allen belt in the real earth magnetotail located at a distance of $2 \mathrm{R}_{\mathrm{E}}$. Physically, the light emission in such images is predominantly attributed to recombination and continuum radiation produced by the plasma. Luminosity variations in the photographs are due mostly to differences in the local plasma density, plasma temperature, and to the visible light of different wavelengths. However, it is difficult to separate one parameter from others (Karl et al. 2002; Bogaerts 2007; Proxauf et al. 2014), and we didn't detect the spectrum of the light emission from the argon plasma. Therefore in each photograph shot, the brighter areas can't be simplified as high density or high temperature areas.

We calculated the plasma confinement time of the plasma current in the magnetotail using the Bohm diffusion time, $t_{B}$. The Bohm magnetic field diffusion coefficient, $D_{B}$, is given by

$\mathrm{D}_{\mathrm{B}}=\mathrm{kT}_{\mathrm{e}} / 16 \mathrm{eB}$

and the plasma confinement time, $\mathrm{t}_{\mathrm{B}}$, is expressed as

$\mathrm{t}_{\mathrm{B}}=\mathrm{R}^{2} / 2 \mathrm{D}_{\mathrm{B}}$

where $\mathrm{R}$ is the effective radius of the plasma column.

We calculate the confinement time for northward IMF conditions according to the previous measurement (Yur et al. 1999). The effective radius of the simulated ring current 
is regarded as about $2 \mathrm{~cm}$. The magnetic field of $20 \mathrm{kG}$ at the equator with the electron temperature, $T_{e}=46 \mathrm{eV}$ and an effective radius, $R=2 \mathrm{~cm}$, the value of Bohm magnetic field diffusion coefficient, $\mathrm{D}_{\mathrm{B}}$ can be calculated as $1.4 \times$ $10^{4} \mathrm{~cm}^{2} \mathrm{~s}^{-1}$, and the plasma confinement time is calculated as $17 \mu \mathrm{s}$. For every photographic shot, the exposure time is $5 \mu \mathrm{s}$. The global structure persists up to $40 \mu \mathrm{s}$. For the northward IMF condition, the images of the radiation belts can be seen, and the inner and outer region can be also observed. The luminosity is higher in the inner region because the confined plasma interacted with the surface of terrella. However, the different two regions are due to the same plasma confinement mechanics, which is different from the radiation in real Van-Allen Belt. When the IMF is southward, the confinement time is about $7 \mu \mathrm{s}$. The shorter confinement time dues to the less weak magnetic field and higher temperature, and only the inner radiation belt near the equator region can be seen sometimes. The plasma confinement was also discussed in other terrella laboratory simulations (Mauel et al. 1992; Rana et al. 2004), and it's the first time that the difference controlled by IMFs was observed.

The global images of laboratory simulation are compared with the computer simulated OpenGGCM model on CCMC. The model is available for input the simulated solar data or real events. In our simulated solar wind data, the plasma density no is $3.29 \times 10^{13} \mathrm{~cm}^{-3}$ and the flow velocity $\mathrm{v}_{\mathrm{o}}$ is $1.1 \times 10^{7} \mathrm{~cm} \mathrm{~s}^{-1}$ for $\mathrm{B}_{\mathrm{o}}=400 \mathrm{G}$. According to Yur et al. (1995), the simulated IMF $B_{p}$ (inside the flowing plasma) in vicinity of the terrella becomes $340 \mathrm{G}$ for $\mathrm{B}_{\mathrm{o}}=400 \mathrm{G}$. With the scaling law by Yur et al. (1995), the simulated IMF $\mathrm{B}_{\mathrm{p}}=340 \mathrm{G}$ corresponds to the IMF $\mathrm{B}_{\mathrm{z}}=34 \mathrm{nT}$ and the no corresponds to $40 \mathrm{~cm}^{-3}$ in the vicinity of the Earth's magnetosphere.

Although all the models in CCMC based on solving the MHD equations and in the magnetosphere and the same electrostatic potential equation in the ionosphere, there are still some differences. On the results for empirical parameters and real events, there is no absolute leader or a perfect one (Gordeev et al. 2015). We choose the OpenGGCM model to obtain the magnetic field lines and temperature profiles in noon-midnight meridian plane. OpenGGCM model solves the MHD equations in a semi-conservative form, and a resistive term is included in the equation for the electric field. After decades of improvements, the model can show good agreement with some in situ observations: the plasma density, temperature and velocity in the magnetotail, interaction between solar wind and the Earth dipole field, the magnetic field in most of the magnetosphere (Wang et al. 2004; Raeder 2006; Connor et al. 2014; Li et al. 2017).

The simulated results are shown in Figs. 8 and 9. For the tilt angle 0 degree, both southward and northward IMFs cases show up and down symmetric in the global structures in Fig. 8. In Fig. 8a, the X-type front side reconnection and tail reconnection can be shown in the southward IMF re- sults that are consistent with the experimental results. The magnetic flux of the southward IMF becomes denser than northward IMF and X-type reconnection location is closed to $40 R_{E}$ in the magnetotail. In Fig. 8b, the closed field lines and comet like tail can be shown in the northward IMF result. For the tilt angle 20 degree, the southward IMF result is shown in Fig. 9a. X type front side magnetic reconnection and the twisted magnetotail can be shown, which are consistent with the experimental results. The magnetotail shape is fat and X-point location is closed to the earth at $30 \mathrm{R}_{\mathrm{E}}$. The northward IMF result is shown in Fig. 9b. Closed field lines can be shown at the tail region. The comet like and the twisted tail due to the tilt is apparent. Since the dipole tilt, the north-south asymmetry occurs, and the neutral sheet falls in the meridian plane which connects the sun with the earth, it would be paralleled for the direction of flow of solar wind in the tail. Topologically, the model results match the laboratory simulation results under southward and northward IMFs. On the other hand, temperature is higher at the tail region when IMF is southward, which also quantitatively matches the previous measurement in the laboratory simulations (Yur et al. 1999). The model simulation results might confirm that the apparent tail region caused by the higher temperature when IMF is southward. When IMF is northward, only a weakly bright area near the equator in the tail region can be seen in the laboratory simulation, which also matches the model simulation.

\section{CONCLUSION}

In series of UCR-T1 space simulation experiments since 1982 (Baum and Bratenahl 1982), the interaction between the solar wind and the earth's magnetosphere have been studied. Under suitable scaling laws, the qualitative structure and quantitative measurements of the parameters of the terrella magnetosphere have been also obtained. The global images of magnetosphere controlled by different IMFs are presented both in $\mathrm{x}-\mathrm{Z}$ and $\mathrm{x}-\mathrm{y}$ plane simultaneously. And the information of the aurora and radiation belt is observed for the first time in the UCR-T1 experiments. The topology and the flank position of the Earth's magnetopause have been investigated in previous paper (Yur et al. 1995, 1999, 2012). The laboratory simulation of magnetosphere for dipole tilts is also a first trial in UCR-T1 experiments. These global images are compared with the computer simulated OpenGGCM model on CCMC.

All such results of the laboratory simulation experiment are difficult to interpret completely, and experiments are never able to scale all the parameters needed to properly simulate the Earth's magnetosphere but all the results of experiment are real. That is why such experiments measurements compare with computer simulation model will be interested.

Unfortunately all budget ended of UCR-T1 experiments in 2002, the all final experimental results are presented in 
(a)

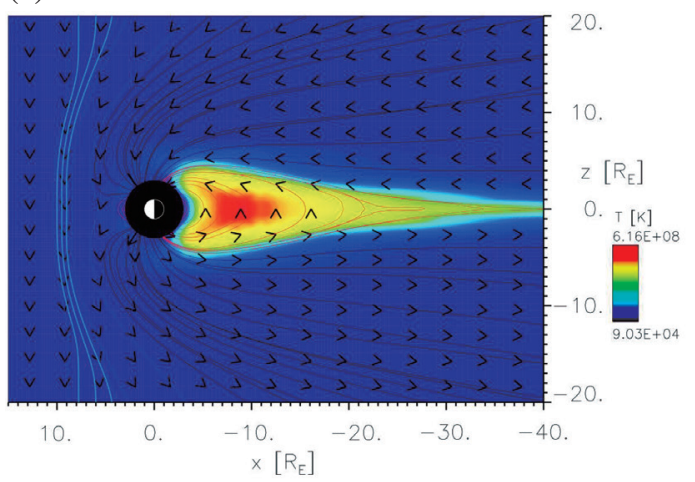

(b)

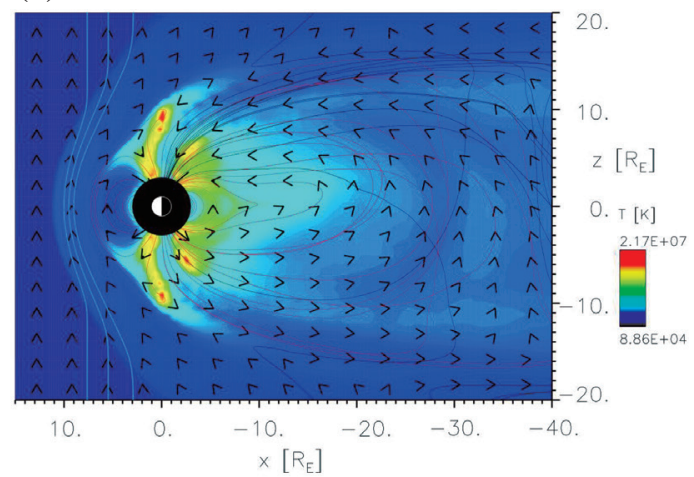

Fig. 8. (a) Computer simulated magnetosphere and temperature profile of OpenGGCM model for southward IMF with dipole tilt 0 degree. (b) Computer simulated magnetosphere and temperature profile of OpenGGCM model for northward IMF with dipole tilt 0 degree.

(a)

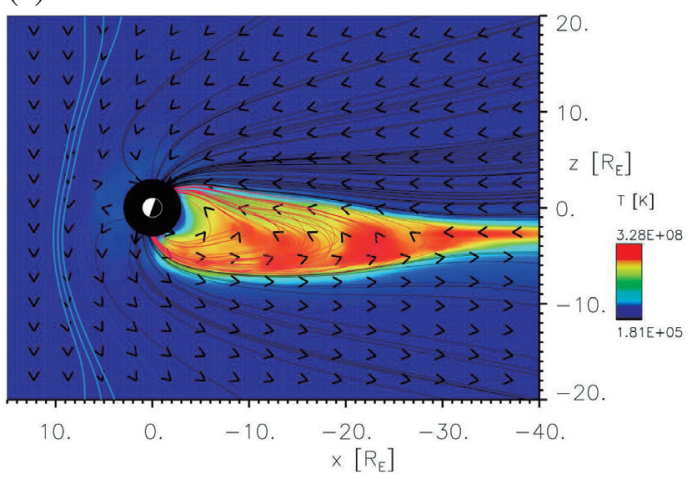

(b)

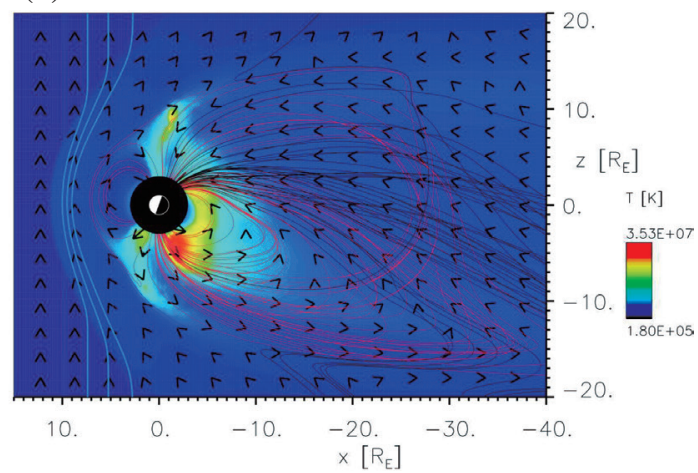

Fig. 9. (a) Computer simulated magnetosphere and temperature profile of OpenGGCM model for southward IMF with dipole tilt 20 degree. (b) Computer simulated magnetosphere and temperature profile of OpenGGCM model for northward IMF with dipole tilt 20 degree.

this paper. Here we don't claim the exactly scaled laboratory model of the earth's magnetosphere but emphasis provides a reference of guide to the latter relevant experiments and researches.

Acknowledgements The computing simulation results have been provided by the Community Coordinated Modeling Center at Goddard Space Flight Center through their public Runs on Request system (http://ccmc.gsfc.nasa.gov). The OpenGGCM Model was developed by the Joachim Raeder and Timothy Fuller-Rowell at the Space Science Center, University of New Hampshire.

\section{REFERENCES}

Baum, P. J. and A. Bratenahl, 1982: The laboratory magnetosphere. Geophys. Res. Lett., 9, 435-438, doi: 10.1029/GL009i004p00435. [Link]

Birkeland, K., 1908: The Norwegian Aurora Polaris Expedition, 1902-1903, Volume I. On the Cause of Magnetic Storms and the Origin of Terrestrial Magnetism,
H. Aschehoug \& Company.

Birn, J. and M. Hesse, 1996: Details of current disruption and diversion in simulations of magnetotail dynamics. J. Geophys. Res., 101, 15345-15358, doi: 10.1029/96JA00887. [Link]

Birn, J., G. Yur, H. U. Rahman, and S. Minami, 1992: On the termination of the closed field line region of the magnetotail. J. Geophys. Res., 97, 14833-14840, doi: 10.1029/92JA01145. [Link]

Birn, J., M. Hesse, and K. Schindler, 1997: Theory of magnetic reconnection in three dimensions. Adv. Space Res., 19, 1763-1771, doi: 10.1016/S02731177(97)00072-0. [Link]

Bogaerts, A., 2007: Plasma diagnostics and numerical simulations: Insight into the heart of analytical glow discharges. J. Anal. At. Spectrom., 22, 13-40, doi: 10.1039/b611436a. [Link]

Bostick, W. H., H. Byfield, and M. Brettschneider, 1963: Plasma flow around a three-dimensional dipole. $J$. Geophys. Res., 68, 5315-5317, doi: 10.1029/j.21562202.1963.tb00011.x. [Link] 
Brady, P., T. Ditmire, W. Horton, M. L. Mays, and Y. Zakharov, 2009: Laboratory experiments simulating solar wind driven magnetospheres. Phys. Plasmas, 16, 043112, doi: 10.1063/1.3085786. [Link]

Cladis, J. B., T. D. Miller, and J. R. Baskett, 1964: Interaction of a supersonic plasma stream with a dipole magnetic field. J. Geophys. Res., 69, 2257-2272, doi: 10.1029/JZ069i011p02257. [Link]

Connor, H.K.,E.Zesta, D. M. Ober, and J.Raeder, 2014: The relation between transpolar potential and reconnection rates during sudden enhancement of solar wind dynamic pressure: OpenGGCM-CTIM results. J. Geophys. Res., 119, 3411-3429, doi: 10.1002/2013JA019728. [Link]

Craven, J. D., J. S. Murphree, L. A. Frank, and L. L. Cogger, 1991: Simultaneous optical observations of transpolar arcs in the two polar caps. Geophys. Res. Lett., 18, 2297-2300, doi: 10.1029/91GL02308. [Link]

Gordeev, E., V. Sergeev, I. Honkonen, M. Kuznetsova, L. Rastätter, M. Palmroth, P. Janhunen, G. Tóth, J. Lyon, and M. Wiltberger, 2015: Assessing the performance of community-available global MHD models using key system parameters and empirical relationships. Space Weather, 13, 868-884, doi: 10.1002/2015SW001307. [Link]

Horton, W. and C. Chiu, 2004: Laser Z-pinch dipole-target experiments to simulate space physics acceleration processes. Phys. Plasmas, 11, 1645-1654, doi: 10.1063/1.1666509. [Link]

Horton, W., C. Chiu, T. Ditmire, P. Valanju, R. Presura, V. V. Ivanov, Y. Sentoku, V. I. Sotnikov, A. Esaulov, N. Le Galloudec, T. E. Cowan, and I. Doxas, 2007: Laboratory simulation of magnetospheric plasma shocks. Adv. Space Res., 39, 358-369, doi: 10.1016/j. asr.2005.01.087. [Link]

Janhunen, P., M. Palmroth, T. Laitinen, I. Honkonen, L. Juusola, G. Facskó, and T. I. Pulkkinen, 2012: The GUMICS-4 global MHD magnetosphere-ionosphere coupling simulation. J.Atmos. Sol.-Terr. Phys., 80, 4859, doi: 10.1016/j.jastp.2012.03.006. [Link]

Karl, S., D. Fletcher, G. Dégrez, T. Magin, and M. Playez, 2002: Assessment of Radiative Transport in an Argon Plasma Flow. In: Harris, R. A. (Ed.), Fourth Symposium on Aerothermodynamics for Space Vehicles, European Space Agency, ESA SP-487, 505-511.

Kawashima, N. and N. Fukushima, 1964: Model experiment for the interaction of solar plasma stream and geomagnetic field. Planet. Space Sci., 12, 1187-1201, doi: 10.1016/0032-0633(64)90165-5. [Link]

Kawashima, N. and S. Mori, 1965: Experiment on the intrusion of plasma into a simulated magnetic cavity. Phys. Fluids, 8, 378-383, doi: 10.1063/1.1761232. [Link]

Li, W.-H., J. Raeder, M. F. Thomsen, B. Lavraud, L.-Z. Lü, and E.-W. Liang, 2017: The formation of superdense plasma sheet in association with the IMF turning from northward to southward. J. Geophys. Res., 122, 29362955, doi: 10.1002/2016JA023373. [Link]

Lyon, J. G., J. A. Fedder, and C. M. Mobarry, 2004: The Lyon-Fedder-Mobarry (LFM) global MHD magnetospheric simulation code. J. Atmos. Sol.-Terr.Phys., 66, 1333-1350, doi: 10.1016/j.jastp.2004.03.020. [Link]

Mauel, M. E., H. H. Warren, and A. Hasegawa, 1992: An experiment to measure collisionless radial transport of energetic electrons confined by a dipole magnetic field. IEEE Trans. Plasma Sci., 20, 626-630, doi: 10.1109/27.199503. [Link]

Minami, S. and Y. Takeya, 1985: Flow of artificial plasma in a simulated magnetosphere: Evidence of direct interplanetary magnetic field control of the magnetosphere. J. Geophys. Res., 90, 9503-9518, doi: 10.1029/ JA090iA10p09503. [Link]

Minami, S., A. I. Podgorny, and I. M. Podgorny, 1993: Laboratory evidence of earthward electric field in the magnetotail current Sheet. Geophys. Res. Lett., 20, 9-12, doi: 10.1029/92GL02492. [Link]

Oka, M., J. Birn, M. Battaglia, C. C. Chaston, S. M. Hatch, G. Livadiotis, S. Imada, Y. Miyoshi, M. Kuhar, F. Effenberger, E. Eriksson, Y. V. Khotyaintsev, and A. Retinò, 2018: Electron Power-Law Spectra in Solar and Space Plasmas. Space Sci.Rev., 214, doi: 10.1007/ s11214-018-0515-4. [Link]

Podgorny, I. M., 1976: Laboratory experiments (plasma intrusion into the magnetic field). In: Williams, D. J. (Ed.), Physics of Solar Planetary Environments, American Geophysical Union, 241-254.

Podgorny, I. M. and R. Z. Sagdeev, 1970: Physics of interplanetary plasma and laboratory experiments. Sov. Phys. Usp., 12, 445-462, doi: 10.1070/PU1970v012n04ABEH003754. [Link]

Podgorny, I. M., E. M. Dubinin, and Y. N. Potanin, 1978: The magnetic field on the magnetospheric boundary from laboratory simulation data. Geophys. Res. Lett., 5, 207-210, doi: 10.1029/GL005i003p00207. [Link]

Podgorny, I. M., A. I. Podgorny, S. Minami, and R. Rana, 2003: The mechanism of energy release and fieldaligned current generation during substorms and solar flares. Advances in Polar Upper Atmosphere Research, 17, 77-83.

Powell, K. G., P. L. Roe, T. J. Linde, T. I. Gombosi, and D. L. De Zeeuw, 1999: A solution-adaptive upwind scheme for ideal magnetohydrodynamics. J. Comput. Phys., 154, 284-309, doi: 10.1006/jcph.1999.6299. [Link]

Proxauf, B., S. Öttl, and S. Kimeswenger, 2014: Upgrading electron temperature and electron density diagnostic diagrams of forbidden line emission. Astron. Astrophys., 561, A10, doi: 10.1051/0004-6361/201322581. [Link] 
Raeder, J., 2006: Flux Transfer Events: 1. Generation mechanism for strong southward IMF. Ann. Geophys., 24, 381-392, doi: 10.5194/angeo-24-381-2006. [Link]

Raeder, J., R. L. McPherron, L. A. Frank, S. Kokubun, G. Lu, T. Mukai, W. R. Paterson, J. B. Sigwarth, H. J. Singer, and J. A. Slavin, 2001: Global simulation of the Geospace Environment Modeling substorm challenge event. J. Geophys. Res., 106, 381-395, doi: 10.1029/2000ja000605. [Link]

Rahman, H. U., G. Yur, G. Wong, and R. S. White, 1989: Laboratory simulation of the large-scale Birkeland current system in the polar region with northward interplanetary magnetic field. J. Geophys. Res., 94, 68736878, doi: 10.1029/JA094iA06p06873. [Link]

Rahman,H.U., G. Yur, R.S. White, J. Birn, and F. J. Wessel, 1991: On the influence of the magnetization of a model solar wind on a laboratory magnetosphere. J. Geophys. Res., 96, 7823-7829, doi: 10.1029/90JA01993. [Link]

Rana, R., S. Minami, S. Takechi, A. I. Podgorny, and I. M. Podgorny, 2004: The dynamical behavior of the earth's magnetosphere based on laboratory simulation. Earth Planets Space, 56, 1005-1010, doi: 10.1186/ BF03351798. [Link]

Saitoh, H., Y. Yano, Z. Yoshida, M. Nishiura, J. Morikawa, Y. Kawazura, T. Nogami, and M. Yamasaki, 2015: Measurement of a density profile of a hot-electron plasma in RT-1 with three-chord interferometry. Phys. Plasmas, 22, 024503, doi: 10.1063/1.4908550. [Link]

Shaikhislamov, I. F., Y. P. Zakharov, V. G. Posukh, E. L. Boyarintsev, A. V. Melekhov, V. M. Antonov, and A. G. Ponomarenko, 2011: Laboratory experiment on region-1 field-aligned current and its origin in the lowlatitude boundary layer. Plasma Phys. Control. Fusion, 53, 035017, doi: 10.1088/0741-3335/53/3/035017. [Link]

Shaikhislamov, I. F., Y. P. Zakharov, V. G. Posukh, A. V.
Melekhov, V. M. Antonov, E. L. Boyarintsev, and A. G. Ponomarenko, 2014: Laboratory model of magnetosphere created by strong plasma perturbation with frozen-in magnetic field. Plasma Phys. Control. Fusion, 56, 125007, doi: 10.1088/0741-3335/56/12/125007. [Link]

Song, J. J., F. J. Wessel, G. Yur, H. U. Rahman, N. Rostoker, and R. S. White, 1990: Fast magnetization of a high-to-low-beta plasma beam. Phys. Fluids, 2, 24822486, doi: 10.1063/1.859512. [Link]

Wang, Y. L., J. Raeder, and C. T. Russell, 2004: Plasma depletion layer: Its dependence on solar wind conditions and the Earth dipole tilt. Ann. Geophys., 22, 4273-4290, doi: 10.5194/angeo-22-4273-2004. [Link]

Wessel, F. J., J. H. Song, N. Postoker, G. Yur, and H. U. Rahman, 1990: Fast magnetization of a low to high beta plasma beam. IEEE International Conference on Plasma Science (ICOPS), 1990 Plasma Science IEEE Conference Record - Abstracts, IEEE, doi: 10.1109/ PLASMA.1990.110713. [Link]

Xiao, Q., Z. Wang, X. Wang, C. Xiao, X. Yang, and J. Zheng, 2017: Conceptual design of Dipole Research Experiment (DREX). Plasma Sci. Technol., 19, 035301, doi: 10.1088/2058-6272/19/3/035301. [Link]

Yur, G., H. U. Rahman, J. Birn, F. J. Wessel, and S. Minami, 1995: Laboratory facility for magnetospheric simulation. J. Geophys. Res., 100, 23727-23736, doi: 10.1029/95JA01162. [Link]

Yur, G., T.-F. Chang, H. U. Rahman, J. Birn, and C. K. Chao, 1999: Magnetotail structures in a laboratory magnetosphere. J. Geophys. Res., 104, 14517-14528, doi: 10.1029/98JA02193. [Link]

Yur, G., C.-C. Cheng, C.-K. Chao, J.-K. Chao, and H.-U. Rahman, 2012: Simulation in the front region of the Earth's magnetosphere. Terr. Atmos. Ocean. Sci., 23, 85-94, doi: 10.3319/TAO.2011.06.20.02(AA). [Link] 